\title{
Análisis de cambios en las coberturas y usos de suelo de la cuenca del río Cuale, Jalisco, México
}

\author{
Bartolo Cruz Romero \\ Jorge Téllez López \\ Fátima Maciel Carrillo González \\ Universidad de Guadalajara (México). Centro Universitario de La Costa. Laboratorio de \\ Ecología, Paisaje y Sociedad \\ cruzromerobartolo@gmail.com \\ jorgetellezlopez@gmail.com \\ fmacielux@gmail.com
}

Recibido: junio de 2019

Aceptado: julio de 2019

Publicado: octubre de 2020

\section{Resumen}

Se clasificó la cobertura de tres imágenes satelitales Landsat (P030 y R046) de los años 1990 (Sensor TM), 2003 y 2013 (Sensor ETM) con el software SIG Arc Gis 10 (ESRI, 2008)®. Y se generaron mapas temáticos con cuatro clases de cobertura, con una fiabilidad del 75\%, el 78\% y el 86\%, y estimaciones del coeficiente Kappa de 0,61, 0,67 y 0,80, respectivamente. Estos mapas fueron comparados mediante matrices de error, donde se registraron procesos evidentes, como el cambio de cobertura en espacios destinados a actividades agrícolas. La tasa de cambio fue del 0,99\% para el bosque templado de coníferas y latifoliadas; el bosque tropical subcaducifolio registró el 1,02\%, y las zonas agrícolas, el $1,08 \%$, respecto a la superficie total de la cuenca. Las zonas urbanas no mostraron cambios en el periodo analizado, y se considera que esto se debe a las condiciones topográficas de la cuenca del río Cuale.

Palabras clave: coberturas; uso de suelo; paisaje; actividades antropogénicas

Resum. Análisi de canvis en les cobertures i usos del sòl de la conca del riu Cuale, Jalisco, Mèxic

Es va classificar la cobertura de tres imatges satel litàries Landsat (P030 y R046) dels anys 1990 (Sensor TM), 2003 i 2013 (Sensor ETM) amb el programari SIG Arc Gis 10 (ESRI, 2008) ®. Es van generar mapes temàtics amb quatre classes de cobertura, amb una fiabilitat del 75\%, el 78\% i el 86\%, i estimacions del coeficient Kappa de 0,61, 0,67 i 0,80 , respectivament. Aquests mapes van ser comparats mitjançant matrius d'error, on es van registrar processos evidents, com el canvi de cobertura en espais destinats a activitats agrícoles. La taxa de canvi va ser del 0,99\% per al bosc temperat de coníferes i latifoliades, el bosc tropical subcaducifolia va registrar l'1,02\%, i les zones agrícoles, l'1,08\%, respecte a la superfície total de la conca. Les zones urbanes no van mostrar canvis en el període analitzat, i es considera que això és degut a les condicions topogràfiques de la conca del riu Cuale.

Paraules clau: cobertures; ús del sòl; paisatge; activitats antropogèniques 
Résumé. Analyse des changements dans la couverture des terres et l'utilisation des terres dans le bassin de la rivière Cuale, Jalisco, Mexique

Capture de trois images satellites Landsat (P030 et R046) des années 1990 (Sensor TM), 2003 et 2013 (Sensor ETM) réalisées à partir du logiciel GIS Arc GIS 10 (ESRI, 2008) §. Quatre cartes ont été générées avec une fiabilité de 75,78 et $86 \%$ le tout en utilisant l'estimation du coefficient Kappa de 0,61, 0,67 et 0,80. Ces cartes ont été comparées avec un programme permettant de détecter des changements et de prendre connaissance des modifications des espaces destinés aux activités agricoles. Les pourcentages des changements sont de $0,99 \%$ pour les forêts de coniferes et de feuillus en zone tempérée. Pour la forêt subtropicale nous avons constaté la perte des feuilles pour certaines espèces, le pourcentage obtenu est de 1,02\% pour les zones agricoles et de 1,08\% pour le bassin. Les zones urbaines n'ont pas démontré de changements significatifs. Nous considérons que cela est dû aux conditions topographiques de la rivière Cuale.

Mots-clés: couverture; utilisation des terres; paysage; activités anthropiques

Abstract. Analysis of land cover and land use changes in the Cuale River basin, Jalisco, Mexico

The coverage of three Landsat satellite images (P030 and R046) from the 1990s (Sensor TM), 2003 and 2013 (Sensor ETM) were classified using ArcGIS 10 software (ESRI, 2008). Thematic maps were generated with four kinds of coverage. The reliability was $75 \%, 78 \%$ and $86 \%$ with Kappa coefficient estimates of $0.61,0.67$ and 0.80 , respectively. The maps were compared using error matrices where evident processes were recorded such as the change in coverage to agricultural areas. The rate of change was $0.99 \%$ for temperate conifer forest cover, $1.02 \%$ for broad-leaved deciduous rainforest cover and $1.08 \%$ for agricultural areas with respect to the total watershed area. Urban areas were unchanged in the period under review likely due to the topographical conditions of the Cuale River basin. Keywords: coverage; land use; landscape; anthropogenic activities

\section{Sumario}
1. Introducción
5. Resultados
2. Antecedentes
6. Discusiones
3. Área de estudio
7. Conclusiones
4. Metodología
Referencias bibliográficas

\section{Introducción}

Las coberturas y los usos de suelo son de gran importancia en la configuración del paisaje (Pickett y Cadenasso, 2013; Armentereas y Vargas, 2016). Schawe et al. (2010) y González et al. (2016) mencionan que existe una relación entre las coberturas y los procesos relacionados con el escurrimiento superficial, ya que la remoción de la vegetación incrementa los procesos de erosión. De tal forma que los cambios de cobertura pueden constituir información cuantitativa y cualitativa importante, que, una vez representada en mapas y datos 
estadísticos, servirá como herramienta valiosa para plantear proyectos de conservación en zonas intervenidas y deterioradas principalmente por actividades antropogénicas. Además, su determinación corresponde a los resultados más visibles de las modificaciones del hombre sobre los ecosistemas (Weng y Wei, 2003). En la cuenca del río Cuale, las coberturas vegetales y los usos de suelo han sufrido diferentes cambios a través del tiempo. Por este motivo, se realizó el presente trabajo, que contribuye al conocimiento de la estimación del cambio producido en las diferentes clases de uso y ocupación de la tierra, con la finalidad de contribuir como insumo para elaborar los planes de ordenamiento ecológico de esta región.

\section{Antecedentes}

De todos los ecosistemas existentes en la Tierra, los bosques son los que soportan mayor presión por las actividades antropogénicas (FAO, 2002; 2007), lo que ha provocado su perturbación parcial o total por el proceso de la deforestación, definida como la pérdida permanente de la cobertura forestal para ser transformada a otros usos de suelo, tales como la agricultura, pastizales, asentamientos humanos e infraestructura (FAO, 2002). Se calcula que hace unos ocho mil años la cobertura de los bosques era de seis mil millones de hectáreas, el doble que en la actualidad (FAO, 2018). No obstante, el deterioro de los bosques fue más severo durante el último siglo, ya que se estima que en este periodo desapareció más cantidad de superficie forestal que durante toda la historia del hombre sobre la Tierra (Lambin y Ehrlich, 1997; Achard et al., 1998). Hoy en día, la deforestación y el cambio de coberturas y usos de suelo son algunos de los problemas ambientales más importantes a los que se enfrenta el mundo. Esto es debido a que presentan serias consecuencias económicas y sociales de largo plazo, ya que están teniendo un efecto negativo en la dinámica ambiental de la Tierra por su repercusión en el calentamiento atmosférico global (Lambin y Ehrlich, 2001). En México, se trata de un problema que se ha presentado desde tiempos precolombinos, sin embargo, durante las últimas cinco décadas, este proceso se ha incrementado de forma espectacular y ha dejado un panorama poco alentador. Los 52 millones de hectáreas de bosques y selvas con que contaba el país en 2000 presentaron de promedio una tasa de deforestación de 631 mil ha/año (FAO, 2007). Esta última cifra puede diferir de la reportada por otras instituciones o investigadores (Velázquez et al., 2002; Semarnat, 2005). Sin embargo, se sitúa entre los rangos extremos reportados para México por la Semarnat (2005) (316-800 mil ha/año) y la Conafor (2015) (253 mil ha/año). Estudios de caso regionales conducidos con diferentes técnicas y métodos han señalado tasas de deforestación entre el 1\% y el $8 \%$ anual, según la región, el tipo de vegetación y el periodo estudiado (Dirzo y García, 1992; Trejo y Hernández, 1996; Mas et al., 2009).

Los datos mencionados han situado los procesos de cambio de cobertura y uso del suelo en el centro de la investigación ambiental actual, ya que constituyen un punto de importancia en los ámbitos gubernamental, académico 
y social (García y Mas, 2008), donde el análisis del cambio de cobertura y uso del suelo representa un medio para entender los mecanismos de este proceso de deterioro, así como el comportamiento hidrológico en cuencas hidrográficas. Este análisis de cambios constituye una guía útil para la toma razonable de decisiones sobre el uso del territorio, que en el país y en el estado han sido aplicadas por diferentes investigadores con fines de monitoreo de hábitat y áreas de protección incorporadas en la caracterización del paisaje, o como información base para instrumentos de planeación, como el ordenamiento ecológico y la determinación del impacto ambiental; y de manera más reciente con los estudios relacionados con el cambio climático (Márquez, 2008; Cabral y Zamyatin, 2009; González et al., 2009; Rosete et al., 2009).

Sin embargo, los estudios de cambio de coberturas y usos del suelo han sido impulsados por una interacción en múltiples escalas espaciales y temporales (Lambin y Ehrlich, 1997). Estas causas suelen depender de las condiciones socioeconómicas mediadas por factores institucionales que en la mayoría de los casos se ven influidos por la dinámica global (Lambin y Ehrlich, 1997). La comprensión de las causas de estos cambios en el ámbito local se ha convertido en un asunto crítico en los intentos de evitar la degradación del suelo. Por tal motivo, los procesos que inducen la deforestación en México se están convirtiendo en actividades de investigación muy estudiadas. En este sentido, en Bahía de Banderas, México, Morales et al. (2016) analizaron los cambios de cobertura vegetal de esta región y obtuvieron que el mayor cambio de uso de suelo se registra de las áreas de bosques a zonas agrícolas. En la cuenca del río Cuale, Bauche (2007) realizó un estudio sobre el cambio de uso de suelo y las posibles causas que han incidido en la pérdida de cobertura vegetal en un periodo aproximado de 30 años. Al mismo tiempo, considera que el pago por servicios ambientales en esta zona puede ser una buena opción para la protección de la cobertura vegetal de toda la cuenca hidrográfica.

\section{3. Área de estudio}

La cuenca del río Cuale se ubica en la costa oeste de México, en el estado de Jalisco (figura 1), en la región donde confluyen la cordillera del Eje Transversal Neovolcánico y la Sierra Madre del Sur (Quintero, 2004). Presenta regiones altitudinales que se distribuyen desde la costa hasta los $2.400 \mathrm{~m} \mathrm{~s}$. n. m. en el área montañosa. Comprende una superficie de 26.993,82 ha. Se localiza en las siguientes coordenadas geográficas: latitud norte entre $20^{\circ} 31^{\prime} 53^{\prime \prime}$ y $20^{\circ} 34^{\prime} 11^{\prime \prime}$, y longitud oeste entre $105^{\circ} 06^{\prime} 53^{\prime \prime}$ y $105^{\circ} 10^{\prime} 05^{\prime \prime}$ hacia el sureste de la ciudad de Puerto Vallarta, Jalisco. Pertenece a la Región Hidrológica Huicicila, se compone de ríos tributarios que son los afluentes del río principal llamado Cuale. El promedio de precipitación anual es de $1.632,2 \mathrm{~mm}$ en las zonas bajas, con un clima sub-húmedo semitropical, y de $1.447 \mathrm{~mm}$ en las partes altas, donde se presenta un clima templado subhúmedo (Conabio, 2008). El entorno geológico está formado por rocas clásticas o detríticas de origen continental. Se aprecia la arenisca de grano fino con fragmentos de roca 
Figura 1. Ubicación de la cuenca del río Cuale, Jalisco, México. Escala 1:50,000. Coordenadas Geográficas. Huso: (UTC-6). Proyección: WGS_1984_UTM_Zone_13N

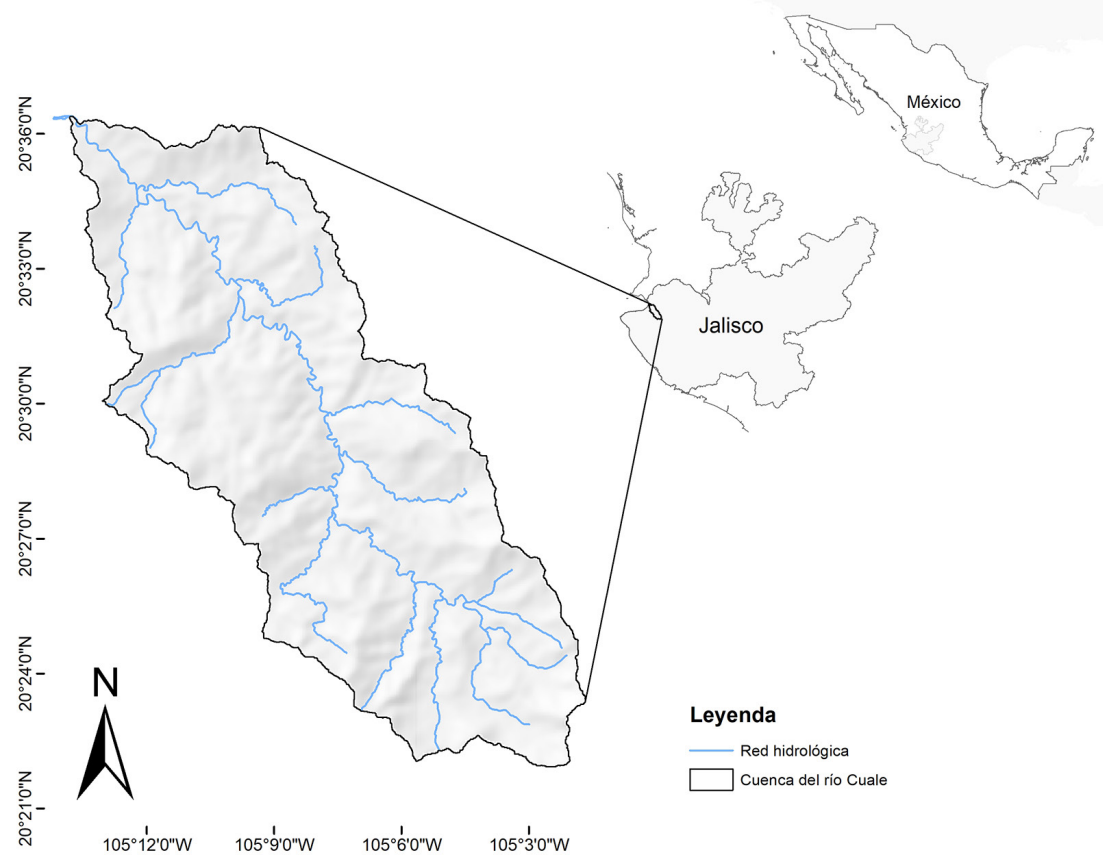

Fuente: elaboración propia.

volcánica y algunos horizontes de arcilla (INEGI, 2010). Los tipos de suelo dominantes corresponden a Regosol eútrico y Litosol (INEGI, 1974). Respecto a la vegetación, la cuenca se encuentra en una zona de transición biogeográfica, característica que da lugar a diversas condiciones ambientales de importancia ecológica debido a su alta biodiversidad y a la presencia de especies endémicas como Aechmea tuitensis y Quercus cualensis. Los tipos de vegetación registrados corresponden a agricultura de pastoral con predominancia de sorgo y maíz. El bosque tropical subcaducifolio está representado cerca de la costa por especies como Brosimum alicastrum, Bursera arborea, Enterolobium ciclocarpum, Ficus sp, Hura polyandra, Tabebuia donell-smithii, Tabebuia rosea, Switenia humilis. A menos de $300 \mathrm{~m}$ de altitud, se registra Attalea cohune, Cordia elaeagnoides e Hymenaea courbaril. El bosque templado de coníferas y latifoliadas predomina en altitudes de hasta $1.420 \mathrm{msnmm}$, y se encuentra un solo estrato arbóreo y un herbáceo de gran desarrollo. Las especies características son Pinus jaliscana, Pinus maximinoi, Quercus aristata, Q. eliptica, Q. magnoliifolia y Q. salicifolia.

Las actividades socioeconómicas de la cuenca del río Cuale se pueden referir al hecho de que en esta área convergen particularidades del ámbito rural y del 
urbano. En la parte baja, el municipio de Puerto Vallarta es el lugar con mayor crecimiento demográfico en los últimos 20 años, con 255.681 habitantes, en comparación con los municipios de Talpa de Allende, con 4.410 habitantes, y Mascota, con 14.245 habitantes (INEGI, 2010). Se considera, por lo tanto, que la cuenca del río Cuale posee una población predominantemente urbana asentada en la parte baja, en la ciudad turística de Puerto Vallarta, pero su base económica en la parte media y alta es agrícola. Por otro lado, es importante señalar que los municipios de Talpa de Allende y Mascota, en su evolución demográfica, no registran incrementos significativos en el tamaño de su población.

\section{Metodología}

Para realizar los mapas de clasificación de coberturas de los años 1990, 2003 y 2013, se utilizaron imágenes de satélite de la plataforma Landsat, tomadas en la época de secas los días 10 de febrero de 1990, con el sensor TM, 13 de mayo de 2003 y 8 de mayo de 2013, ambas con el sensor ETM. Posteriormente, se delimitó el área de estudio y se llevó a cabo el procesado multiespectral con ayuda del software SIG Arc Gis 10 (ESRI, 2008) ${ }^{\circledR}$ y sus herramientas. Luego se realizaron los tratamientos digitales de las imágenes, seguidos de un análisis visual que permitió disponer de las primeras interpretaciones de las coberturas. Este proceso tuvo como primer paso la composición en falso color, utilizando las bandas 2, 3 y 4 de las tres imágenes de satélite. La finalidad fue representar los tres colores básicos de visualización: rojo, verde y azul (red, green, blue: $R G B$ ) para poder resaltar las coberturas que presentan mayor reflectividad.

La metodología utilizada para la elaboración de los mapas finales consistió en realizar una clasificación supervisada en las imágenes, y el algoritmo empleado fue el de máxima verosimilitud (maximum likelihood algorithm), el cual considera que los datos asumen una distribución normal o gaussiana. Esto permite describir cada categoría a través de una función de probabilidad, a partir de su vector de las medias y la matriz varianza-covarianza. Chuvieco (2010) recomienda este método cuando existe un conocimiento previo del área, ya que permite que el analista seleccione las clases de cobertura de acuerdo con sus objetivos. En este caso, se consideraron clases de cobertura mixtas, debido a que en sitios con pendientes pronunciadas se presentaban con un tipo de distribución espacial combinada y muy estrecha, y resultaba impráctica su delimitación individual. Esta combinación incluyó clases de cobertura donde una predominó sobre la otra. La valoración de las proporciones se realizó a través de estimaciones visuales del intérprete. Para este caso, se utilizaron las coberturas definidas en la tabla 1 .

De acuerdo con Challenger y Soberon (2008) e INEGI (2008) y considerando la heterogeneidad de la vegetación en México, las coberturas vegetales se agruparon en un sistema de clasificación jerárquico que combina el nombre de la formación y el tipo de comunidad. Fueron establecidas teniendo como base información previa de campo georreferenciada (puntos 
Tabla 1. Clases utilizadas en la clasificación de las imágenes de satélite de los años 1990, 2003 y 2013

\begin{tabular}{llc}
\hline Clases & Descripción & Identificador \\
\hline Zonas urbanas & Comprende las áreas con infraestructura urbana. & 1 \\
\hline $\begin{array}{l}\text { Bosque tropical } \\
\text { subcaducifolio }\end{array}$ & $\begin{array}{l}\text { Compuesto por numerosas especies de gran altura, } \\
\text { de 10 a 40 m. Destacan especies arbóreas de los géneros } \\
\text { Brosimum, Cedrela, Sideroxylon, Trichilia, Sapium, } \\
\text { Dendropanax, Bursera y Ficus. }\end{array}$ & 2 \\
\hline Zonas agrícolas & $\begin{array}{l}\text { Áreas donde se llevan a cabo actividades agrícolas: } \\
\text { cultivos, pecuarias, rosa-quema, suelo expuesto y pastos. }\end{array}$ & 3 \\
\hline $\begin{array}{l}\text { Bosque templado } \\
\text { de coníferas y }\end{array}$ & $\begin{array}{l}\text { Comunidad de aspecto siempre verde donde coexisten } \\
\text { latifoliadas }\end{array}$ & $\begin{array}{l}\text { esta coexistencia es favorecida por las actividades humanas. } \\
\text { Los principales componentes arbóreos corresponden a los }\end{array}$ \\
& $\begin{array}{l}\text { géneros Pinus, Quercus, Magnolia, llex, Cornus, Tilia, } \\
\text { Dendropanax, Carpinus, Ostrya, Fraxinus, Styrax y Saurauia. }\end{array}$ & \\
\hline
\end{tabular}

Fuente: tomado de Challenger y Soberon (2008).

de control), información documental y material fotográfico obtenido con una cámara digital.

La interpretación visual permitió la obtención de resultados más confiables y el uso de información adicional a la información espectral (mapas existentes, datos de campo, conocimientos y experiencia del intérprete) (Mas et al., 2003).

Posteriormente, se aplicó un filtro para eliminar el efecto pimienta resultante en formato raster, el cual se transformó a archivo vector. Este formato garantiza la precisión en el cálculo de superficies y la eliminación de polígonos denominados islas. Para este caso de estudio, se eliminaron polígonos menores a 20 ha. La fase de comprobación y reclasificación consistió en una confirmación preliminar con información de campo y la selección de puntos críticos que no se ajustaron a la clasificación, con la reasignación de sus áreas por corrección directa o asignación individual de polígonos a una determinada cobertura, siempre comparándola con la imagen original. Para evaluar la imagen clasificada, se utilizó un listado de 92 puntos de verificación obtenidos durante diez salidas de campo con un GPS marca Garmin, siguiendo un tipo de muestreo sistemático no regular alineado según rutas y caminos secundarios. Para cada punto, se registró su cobertura real con resultados provenientes de validación en campo. Con esta información, se generó una tabla que posteriormente se utilizó como matriz de error junto con los puntos deducidos por la clasificación, para evaluar así los conflictos que se presentan entre categorías (Chuvieco, 2010). La matriz de error es bidimensional, donde las filas corresponden a las clases de referencia y las columnas a las categorías deducidas de la interpretación. Lógicamente, ambas tendrán el mismo número y significado. En resumen, es una matriz cuadrada $n \times n$, donde $n$ indica el número de categorías. La diagonal de dicha matriz expresa el número de puntos de verificación donde se produce acuerdo entre las dos fuentes (fotointerpretación y realidad), mientras que los marginales suponen errores de asignación. La relación entre el número 
de puntos correctamente asignados y el total expresa la fiabilidad del mapa. Los residuales en las filas indican tipos de cubierta real que no se incluyeron en el mapa, mientras que los residuales en las columnas implican cubiertas del mapa que no se ajustan a la realidad. De esta manera, representan errores de omisión y de comisión, respectivamente (USDA, 1995). Para determinar la fiabilidad del mapa, se empleó la siguiente fórmula:

$$
F_{m}=\frac{\sum X_{i}}{\sum \sum X_{i j}} \times 100
$$

$F_{m}=$ fiabilidad del mapa, $\sum X_{i}=$ sumatoria de los aciertos o acuerdo observado, $\Sigma \Sigma X_{i i}=$ total de los puntos muestreados

Para analizar las relaciones múltiples entre las distintas clases, se calculó el índice Kappa $(k)$. Considerado como una técnica de análisis categórica multivariante, que evalúa si una matriz de error es significativamente diferente de otra (Congalton y Green, 1999). Y se calculó con la siguiente ecuación:

$$
k=\frac{N \sum X_{i i}-\sum\left(X_{i} \times X_{+i}\right)}{N^{2}-\sum\left(X_{i} \times X_{+i}\right)}
$$

$N=$ total de datos muestreados, $X_{i i}=$ sumatoria de los aciertos o acuerdo observado, $\left(X_{i} X_{+i}\right)=$ concordancia esperada (producto de marginales)

De acuerdo con la técnica anterior, se debe considerar un nivel mínimo de validación cartográfica superior al $75 \%$. En este sentido, es posible afirmar que el mapa fue debidamente validado, y se consideran tres clases de resultados: un valor $k<0,4$ representa una concordancia pobre; un valor entre 0,4 y 0,8 , una concordancia moderada, y un valor mayor de 0,8 una fuerte concordancia en la clasificación real. Por último, para calcular la tasa de cambio de coberturas se utilizó la ecuación formulada por la FAO (2007) expresada de la siguiente forma:

$$
\delta=1-\left[\frac{S 1-S 2}{S 1}\right] 1 / n
$$

$\delta=$ tasa de cambio, $S 1=$ cobertura al inicio del periodo, $S 2=$ cobertura al final del periodo, $n=$ número de años entre las dos fechas

\section{Resultados}

A partir de la comparación de las imágenes clasificadas (figuras 2, 3 y 4), se obtuvieron las áreas de las coberturas y los porcentajes que representan las variaciones de un periodo a otro. El análisis se realizó en una superficie de 
Figura 2. Coberturas y usos de suelo para el año 1990

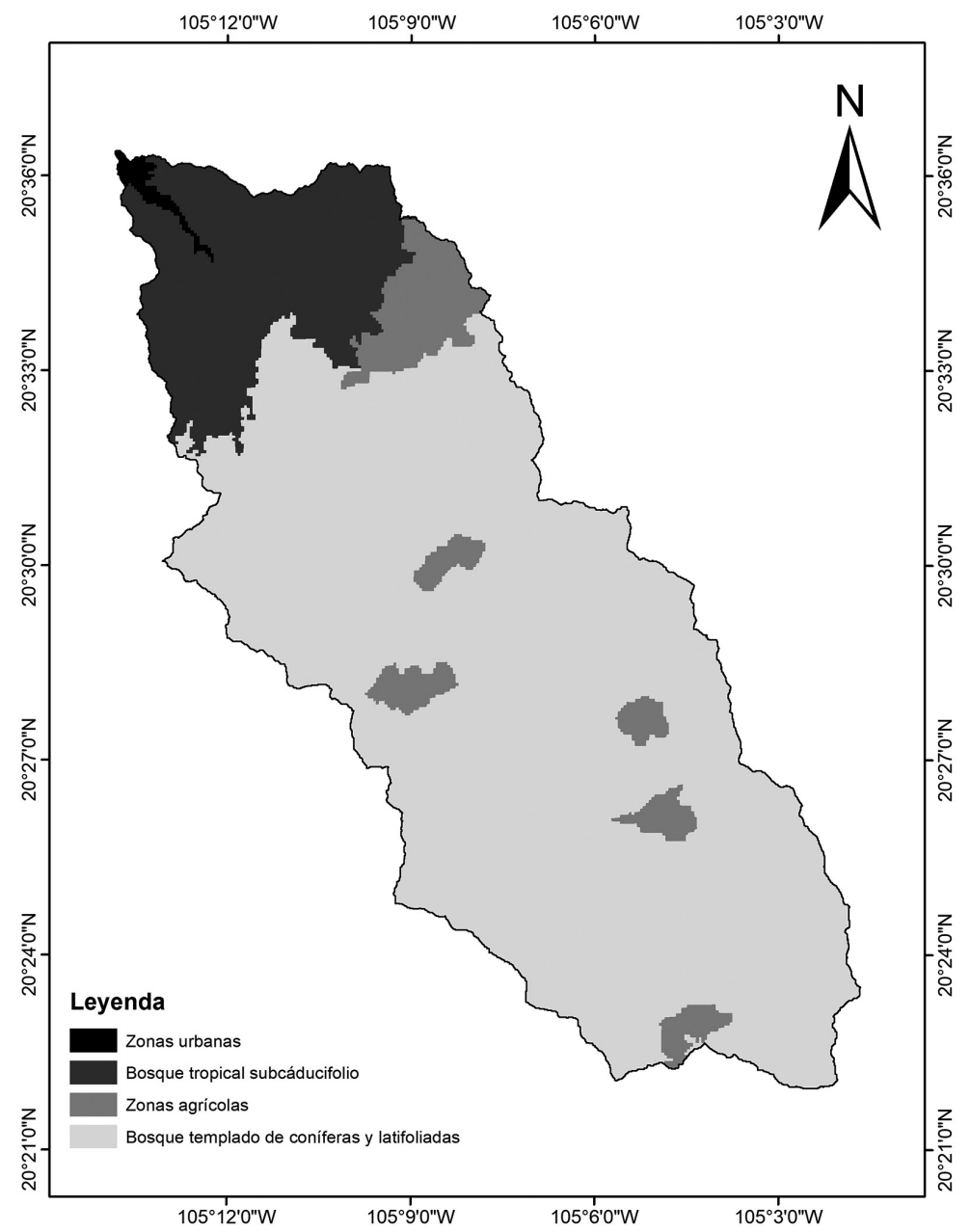

Fuente: elaboración propia.

26.855,57 ha que comprenden la delimitación de la cuenca del río Cuale, y su cálculo presentó un margen de error del 0,53\%, resultado del promedio de las coberturas obtenidas para cada año de estudio. La clasificación supervisada se realizó con el algoritmo de máxima verosimilitud y se generaron tres mapas con cuatro coberturas: zonas urbanas, bosque tropical subcaducifolio, zonas agrícolas y bosque templado de coníferas y latifoliadas. Durante el periodo analizado, el mayor porcentaje de cobertura correspondió al bosque templado de coníferas y latifoliadas, con el $78,4 \%$ en 1990 , el $62,92 \%$ en 2003 y el $56,56 \%$ 
Figura 3. Proyectos de regeneración urbana

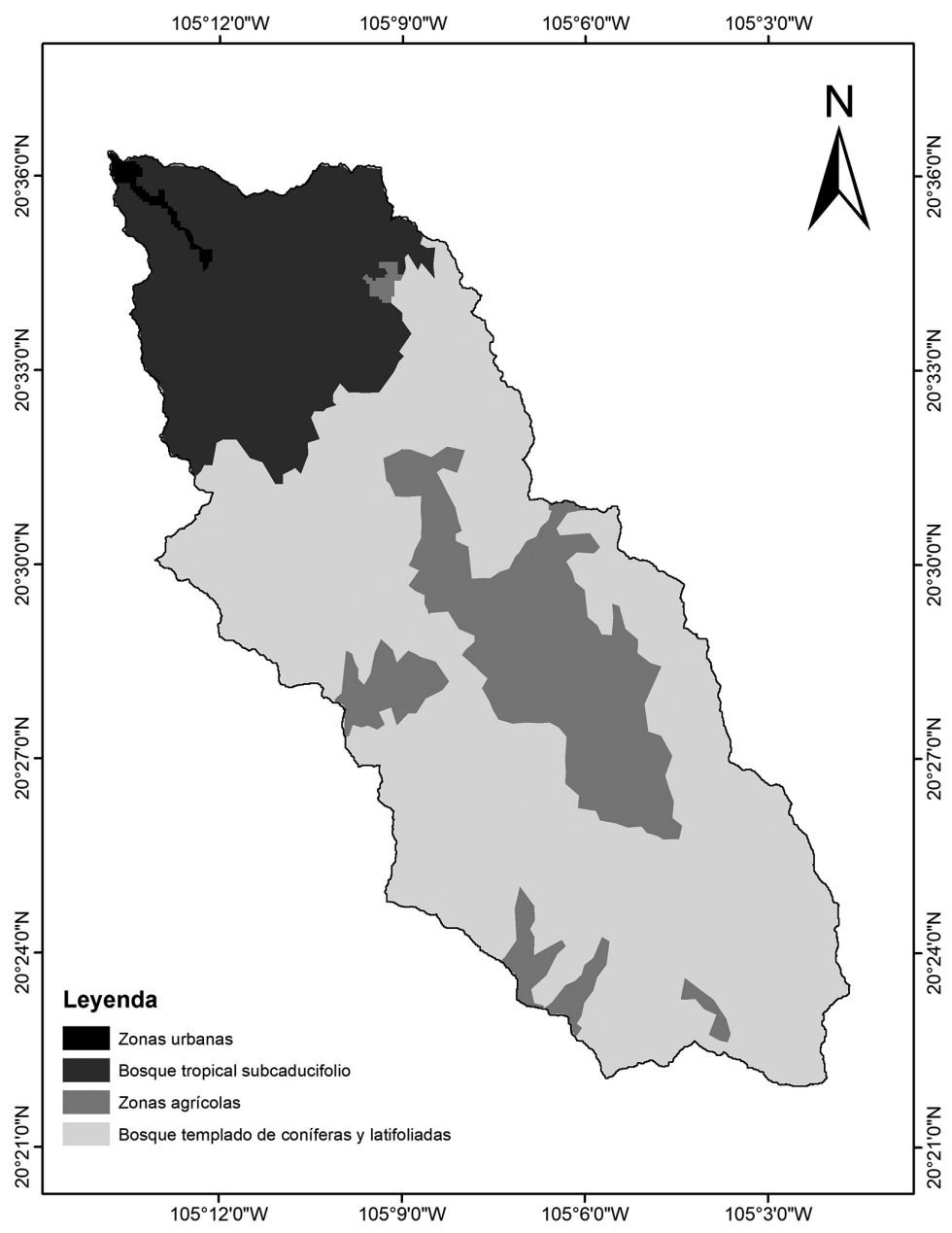

Fuente: elaboración propia.

en 2013, y la tasa de cambio fue del 0,99\%. El bosque tropical subcaducifolio registró el $14,94 \%$ en 1990 , el $20,70 \%$ para 2003 y el $23,63 \%$ en 2013 , y la tasa de cambio fue del $1,02 \%$. La menor cobertura correspondió a las zonas urbanas, con el 0,92\%, sin presentar incremento durante las fechas analizadas. En las tablas 2 y 3 , se observa que las zonas agrícolas son las coberturas que más han aumentado en superficie. En el año 1990 ocupaban 1.606,71 ha $(5,98 \%)$, para 2003 aumentaron a $4.311,49$ ha $(15,97 \%)$ y en 2013 se registraron $5.104,28$ ha $(18.91 \%)$, y la tasa de cambio fue del 1,08\%. La clase más 
Figura 4. Coberturas y usos de suelo para el año 2013

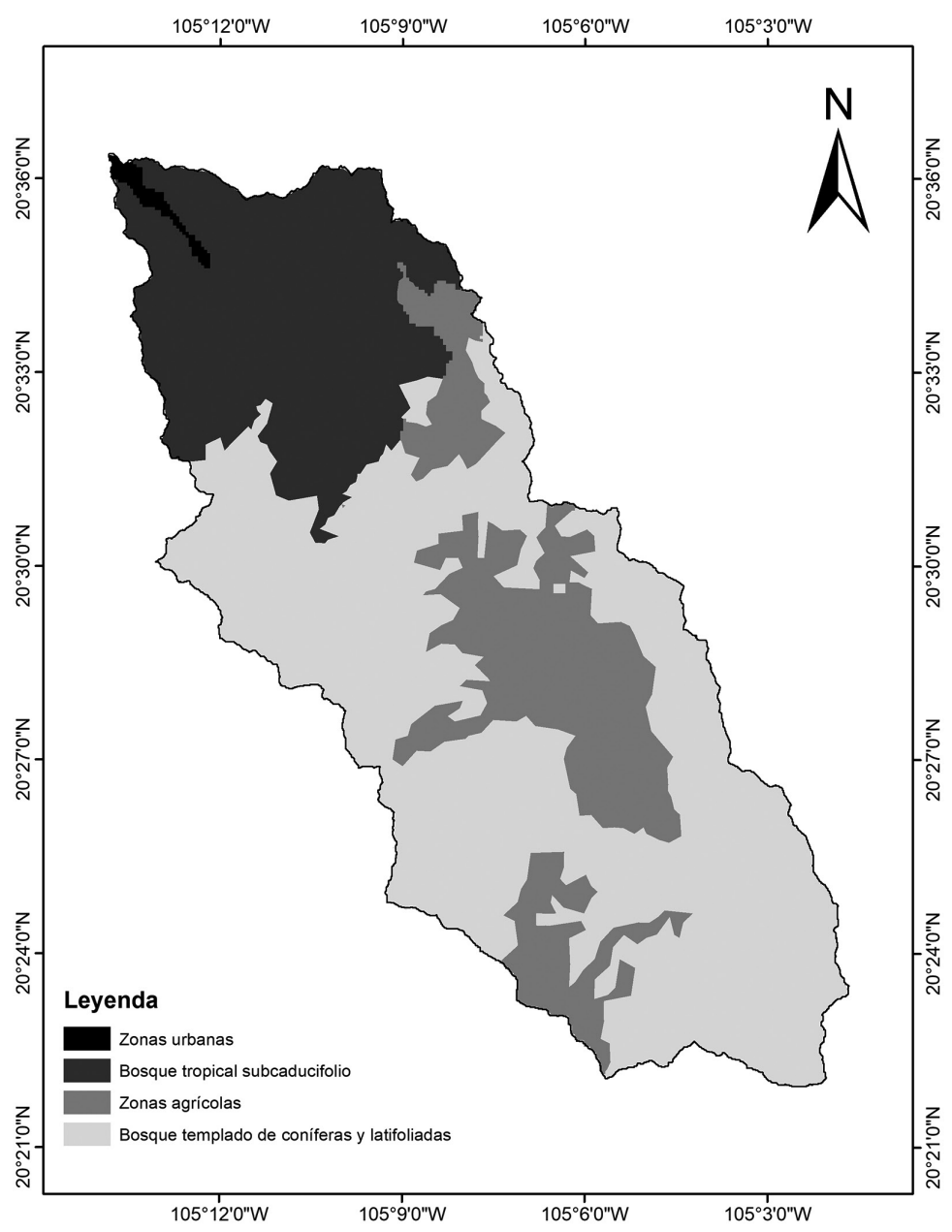

Fuente: elaboración propia.

afectada por estos cambios fue el bosque templado de coníferas y latifoliadas, donde la cobertura ha disminuido de $20.988,92$ ha $(78,14 \%)$ a $15.264,15$ ha $(56,56 \%)$. Estos datos indican que en la superficie que corresponde a esta clase se desarrollan la mayoría de las actividades relacionadas con el sector rural, como el cultivo de temporal, pastoreo y aprovechamiento forestal. Sin embargo, es importante señalar que la actividad turística impulsada desde Puerto Vallarta ha sido generadora de cambios socioeconómicos en la región y ha impactado en las poblaciones de las localidades rurales, principalmente 
Tabla 2. Coberturas y usos de suelo en hectáreas

\begin{tabular}{lrrr}
\hline Clases & \multicolumn{1}{c}{$\mathbf{1 9 9 0}$} & \multicolumn{1}{c}{$\mathbf{2 0 0 3}$} & \multicolumn{1}{c}{$\mathbf{2 0 1 3}$} \\
\hline Zonas urbanas & 248,28 & 248,87 & 248,90 \\
Bosque tropical subcaducifolio & $4.013,43$ & $5.444,73$ & $6.377,88$ \\
Zonas agrícolas & $1.606,71$ & $4.311,49$ & $5.104,28$ \\
Bosque templado de coníferas y latifoliadas & $20.988,92$ & $16.979,09$ & $15.264,15$ \\
Total & $26.855,57$ & $26.855,57$ & $26.855,57$ \\
\hline
\end{tabular}

Fuente: elaboración propia.

Tabla 3. Porcentaje de coberturas y usos de suelo

\begin{tabular}{lrrr}
\hline Clases & 1990 & $\mathbf{2 0 0 3}$ & $\mathbf{2 0 1 3}$ \\
\hline Zonas urbanas & 0,92 & 0,92 & 0,92 \\
Bosque tropical subcaducifolio & 14,87 & 20,17 & 23,63 \\
Zonas agrícolas & 5,95 & 15,97 & 18,91 \\
Bosque templado de coníferas y latifoliadas & 78,14 & 62,92 & 56,56 \\
Total & 100 & 100 & 100 \\
\hline
\end{tabular}

Fuente: elaboración propia.

Tabla 4. Matriz de error para el año 1990

\begin{tabular}{lrrrrc}
\hline Clase & ZU & BTS & ZA & BTCL & Total \\
\hline ZU & 24 & 2 & 0 & 0 & 26 \\
BTS & 4 & 67 & 0 & 0 & 71 \\
ZA & 0 & 0 & 19 & 1 & 20 \\
BTCL & 0 & 7 & 69 & 133 & 209 \\
Total & 28 & 76 & 88 & 134 & 326 \\
\hline
\end{tabular}

Fiabilidad 244/326 = 0,75, 75\%

Índice Kappa = 0,61

Fuente: elaboración propia.

por migración en búsqueda de empleo en la zona turística. Esto ha originado el abandono del campo para integrarse en las actividades económicas dirigidas a la atención del turismo.

En las tablas 4, 5 y 6 se presentan las matrices de error obtenidas con la clasificación de los píxeles para las áreas de entrenamiento. La diagonal indica los píxeles clasificados correctamente. Los demás valores señalan las clases de cobertura asignadas a los píxeles que fueron clasificados incorrectamente. La fiabilidad y el índice Kappa para la evaluación de las firmas espectrales empleadas en las imágenes Landsat TM 1990 fueron del 75\% y el 0,61; en Landsat TM 2003, del 78\% y el 0,67; en Landsat ETM 2013, del 86\% y el 0,80, respectivamente. La fiabilidad y el índice Kappa fueron aceptables en todos los casos $\left(F_{m}>75 \%\right)$ y $(k<0,4)$ (Mas y Pérez, 2003). Los resultados se consideran satisfactorios por la validación mediante puntos de control obtenidos durante las salidas al campo. 
Tabla 5. Matriz de error para el año 2003

\begin{tabular}{lrrrrr}
\hline Clase & ZU & BTS & ZA & BTCL & Total \\
\hline ZU & 22 & 2 & 0 & 0 & 24 \\
BTS & 6 & 70 & 0 & 11 & 87 \\
ZA & 0 & 0 & 46 & 6 & 52 \\
BTCL & 0 & 4 & 42 & 117 & 163 \\
Total & 28 & 76 & 88 & 134 & 326 \\
\hline
\end{tabular}

Fiabilidad 255/326 $=0,78,78 \%$

Índice Kappa =0,67

Fuente: elaboración propia.

Tabla 6. Matriz de error para el año 2013

\begin{tabular}{|c|c|c|c|c|c|}
\hline Clase & $Z U$ & BTS & $Z A$ & BTCL & Tota \\
\hline$\overline{Z U}$ & 19 & 2 & 0 & 0 & 21 \\
\hline BTS & 9 & 69 & 4 & 5 & 87 \\
\hline ZA & 0 & 0 & 72 & 7 & 79 \\
\hline BTCL & 0 & 5 & 12 & 122 & 139 \\
\hline Total & 28 & 76 & 88 & 134 & 326 \\
\hline
\end{tabular}

Fiabilidad $282 / 326=0,86,86 \%$

Índice Kappa $=0,80$

$\mathrm{ZU}=$ zonas urbanas, $\mathrm{BTS}=$ bosque tropical subcaducifolio, $\mathrm{ZA}=$ zonas agrícolas, $\mathrm{BTCL}=$ bosque templado de coníferas y latifoliadas.

Fuente: elaboración propia.

\section{Discusiones}

La clasificación de las imágenes se realizó con cuatro clases generales que conjuntaron coberturas más específicas (tabla 1), debido a que una definición del área de estudio en más clases traería como consecuencia que los datos contenidos en las áreas de entrenamiento de las clases presentaran variabilidad y distribuciones de frecuencia polimodales y restaría eficacia al algoritmo de máxima verosimilitud (Campbell, 2007). Por tal motivo, la categorización correspondiente a zonas urbanas, bosque tropical subcaducifolio, zonas agrícolas y bosque templado de coníferas y latifoliadas generó, en el proceso de agrupación de píxeles, distribuciones con tendencia normal, lo que contribuyó a una mayor eficacia en el proceso de clasificación. Se considera que el $0,53 \%$ de margen de error que se obtuvo en el cálculo de la superficie total de la cuenca se debió a los diversos cambios de altitud dentro de las imágenes clasificadas (Chuvieco, 2010). La imagen del año 1990 fue la que presentó menor fiabilidad del mapa, con un porcentaje del $75 \%$ de píxeles clasificados correctamente; para la imagen de 2003, la fiabilidad fue del 78\%, y para 2013, del $86 \%$. La información anterior corresponde a lo reportado por Rojas y Ortiz (2009) y Weng (2009), quienes mencionan que la clasificación supervisada se fundamenta en el conocimiento previo de las coberturas y los datos de campo 
que se relacionan con cada clase espectral de la imagen. Por otro lado, Mas (1999) menciona que con la técnica de detección de cambios de cobertura se pueden obtener resultados satisfactorios cuando los mapas presentan altos niveles de fiabilidad. Respecto a la tasa de cambio de coberturas, queda claro que la expansión de zonas agrícolas es el principal factor de deforestación que incide en el bosque templado de coníferas y latifoliadas, con una tasa de cambio durante el periodo analizado del $0,99 \%$. Este valor se considera bajo con respecto a los casos de estudio en otras áreas tropicales del país. Para la selva de Los Tuxtlas, se han estimado tasas de deforestación del 4,2\% (Dirzo et al., 1992); en Chiapas, del 1,58 al 2,13\% para Huistán, y del 0,46 al 3,42\% para Chanal (Ochoa y González, 2000). En la selva Lacandona, se reportan promedios que van del 3,31 al 2,14\% (Mendoza y Dirzo, 1999), y el 1,4\% para el estado de Morelos (Trejo y Dirzo, 2000). A escala regional, en la vertiente del Pacífico, se registra una tasa de cambio con un promedio del 5,1\% anual (Berlanga-Robles et al., 2011; Nájera et al., 2010; Osorio et al., 2015; Nene et al., 2017).

Las zonas urbanas fueron coberturas que no registraron cambios en el periodo analizado. Se considera que esto se debe a la baja disponibilidad de terrenos para la construcción de infraestructura urbana en la parte baja de la cuenca y a la influencia de la topografía, ya que aproximadamente 248 ha de superficie en esta zona corresponden a terrenos con pendientes menores a $10^{\circ}$. La mayor parte de la superficie de la cuenca presenta pendientes pronunciadas que incluso llegan a $90^{\circ}$. Con respecto al bosque tropical subcaducifolio, la tasa de cambio fue baja $(1,02 \%)$, valor que refiere un decremento en la deforestación. Se considera que esta tendencia se debe a que el 59\% de la población de la cuenca baja se dedica a actividades relacionadas con el sector económico de servicios, principalmente el turismo (INEGI, 2010). De tal manera que el uso turístico de los recursos naturales de la cuenca recae principalmente en el municipio de Puerto Vallarta, y esta actividad contribuye a impulsar la conservación de los recursos naturales en la región (Cruz et al., 2013). Por otra parte, el uso agrícola es más intenso en la parte alta de la cuenca, donde se localizan algunas comunidades rurales que basan su sustento en esta actividad. De manera general, los resultados de este análisis muestran una tendencia a la degradación de los recursos naturales y un aumento en el uso agrícola del suelo en la parte alta, que corresponde a la advertencia resultante de estudios previos (Ramírez, 2001; Velázquez et al., 2002; Gómez et al., 2006; Reyes et al., 2006; Pinedo et al., 2007). Estos cambios pueden desembocar en una alteración de las condiciones naturales de una cuenca (Barradas et al., 2004). En este sentido, teniendo en cuenta con los cambios ocurridos de las coberturas vegetales naturales a zonas agrícolas, se puede inferir que existe la posibilidad de que se presenten eventos extremos asociados a la deforestación en la parte alta de la cuenca, como pueden ser los efectos del escurrimiento superficial ocasionados por la precipitación, procesos que pueden incrementar el riesgo de erosión por escorrentía. 


\section{Conclusiones}

La clasificación de imágenes registró valores de fiabilidad del 75\%, el 78\% y el $86 \%$, que indican una fuerte coincidencia entre los datos de referencia y los mapas temáticos. Se puede distinguir que la presencia de coberturas de bosques (tropical subcaducifolio, con 6.377,88 ha, y templado de coníferas y latifoliadas, con 15.264,15 ha) es un rasgo distintivo de los recursos naturales de esta región. Además, existe un proceso de cambio de coberturas, más acentuado por el incremento de las zonas agrícolas, con una tasa del 1,08\% en el periodo estudiado. Su avance se extiende hacia las áreas de vegetación original, con el inicio de procesos de tala que derivan en un problema socioambiental relacionado con el uso forestal, ya que esta práctica incide en el entorno biofísico natural de forma negativa y afecta las coberturas forestales en la parte media y alta de la cuenca. Sin embargo, en la parte baja, se tiene el escenario de la zona costera, en la cual las actividades socioeconómicas de los pobladores están basadas en el turismo. Esta situación impulsa el avance de la zona urbana y origina cambios de cobertura en la vegetación del bosque tropical subcaducifolio. Este proceso de emplazamiento urbano deberá ser considerado en los planes de ordenamiento ecológico y territorial, para establecer estrategias de operación que aseguren la conservación y el desarrollo a largo plazo de los sistemas naturales y sociales presentes en la cuenca.

\section{Referencias bibliográficas}

Achard, F.; Eva, H.; Glinni, A.; Mayaux, P.; Richards, T. y Stibig, H. J. (eds.) (1998). Identification of deforestation hot spot areas in the humid tropics, TREES Series B, 4. Luxemburgo: European Commission.

ARMENTERAS, D. y VARGaS, O. (2016). «Patrones del paisaje y escenarios de restauración: acercando escalas». Acta biol. Colomb., 21 (1), 229-239. <http://dx.doi.org/10.15446/abc.v21n1sup.50848>

Barradas, V.; Cervantes, P. y Puchet, A. (2004). «Evidencia de un cambio climático en la región de las grandes montañas del Estado de Veracruz, México». En: García Codron, J. C.; Diego Liaño, C.; Fernández de Arróabe, P.; Garmendia Pedraja, C. y Rasilla Állvarez, D. (eds.). El clima entre el mar y la montaña, Serie A, 213-220. Santander, España: Asociación Española de Climatología y Universidad de Cantabria.

BAUCHE, P. (2007). Interactions of payment for hydrological services and forest transitions: A case study of the Rio Cuale watershed. Montreal, Quebec: Thesis of Master Science, McGill University.

Berlanga Robles, C. A.; Ruiz Luna, A.; Covarrubias Legaspi, O. y HernándezGuZMÁN, R. (2011). «Patrones de escurrimiento en la Cuenca Lechuguilla-OhuiraNavachiste, Sinaloa, asociados a cambios de uso del suelo: Una aproximación con el modelo del número de curva». Boletín de la Sociedad Geológica Mexicana, 63 (1), 39-52.

Cabral, P. y Zamyatin, A. (2009). «Markov Processes in Modeling Land Use and Land Cover Changes in Sintra-Cascais, Portugal». Dina, 76 (158), 191-198.

CampBell, J. B. (2007). Introduction to Remote Sensing. Nueva York: Guilford. 
Challenger, A. y Soberón, J. (2008). «Los ecosistemas terrestres». Capital Natural de México, 87-108. México: Conabio.

Chuvieco, E. (2010). Teledetección ambiental: la observación de la Tierra desde el espacio. Barcelona: Planeta.

CONABIO (2008). «Regiones Terrestres Prioritarias de México». En: ArRIAGA, L.; EsPInOZA, J. M.; Aguilar, C.; MartíneZ, E.; GÓmeZ, L. y LOA, E. (coord). Regiones Terrestres Prioritarias de México. México: Conabio.

CONAFlor (2015). Comisión Nacional Forestal. Programa Nacional Forestal 2014-2015. México: Semarnat.

Congalton, R. G. y Green, K. (1999). Assessing the accuracy of remotely sensed data: principles and practices. Washington, DC: Lewis Publisher.

Cruz Romero, B.; Delgado Quintana, J. A.; Téllez lópez, J. y Carrillo GonZÁLEZ, F. M. (2013). «Análisis socioeconómico de la cuenca del río Cuale, Jalisco, México. Una contribución para la declaración del Área Natural Protegida Reserva de la Biosfera El Cuale». Observatorio Iberoamericano del Desarrollo Local y la Economía Social, 7 (14). Disponible en: <https://www.eumed.net/rev/oidles/14/ rio-cuale.pdf>.

Dirzo, R. y García, M. C. (1992). «Rates of Deforestation in Los Tuxtlas, a Neotropical Area in the Southeast México». Conservation Biology, 6 (1), 84-90.

ESRI (2008). Environmental Systems Research Institut. <www.esri.com>. [Consulta: 9 de abril de 2011].

FAO (2002). State of the world forest 2001. Roma: Food and Agriculture Organization.

- (2007). Situación de los bosques del mundo. <www.fao.org>. [Consulta: 8 de mayo de 2013].

- (2018). El estado de los bosques del mundo. Las vías forestales hacia el desarrollo sostenible. Roma. Licencia: CC BY-NC-SA 3.0 IGO. Disponible en: <http://www. fao.org/3/i9535es/i9535es.pdf>.

GarCía, M. T. y MAS, J. F. (2008). «Comparación de metodologías para el mapeo de la cobertura y uso del suelo en el sureste de México». Investigaciones Geográficas, Boletin del Instituto de Geografia UNAM, 67, 7-19.

Gómez, M.; Vega, P.; Ramírez, E.; Palacio, M. y Galicia, L. (2006). «Projecting land-use change processes in the Sierra Norte of Oaxaca, Mexico». Applied Geography, 26 (3-4), 276-290.

González, S. A.; Bojórquez, S. I.; Nájera, G. O.; García, P. D., Madueño, M. A. y Flores, V. F. (2009). "Regionalización ecológica de la llanura costa norte de Nayarit». Investigaciones Geográficas, Boletín del Instituto de Geografía UNAM (69), 21-32.

GonzÁlez González, A.; Álvarez Figueroa, P.; González Fuertes, M. y Aguirre MendozA, Z. (2016). "Influencia de la cobertura vegetal en los coeficientes de escorrentía de la cuenca del río Catamayo, Ecuador». CEDAMAZ, 98 (1), 50-59. Disponible en: <http://revistas.unl.edu.ec/index.php/cedamaz/article/view/61>.

INEGI (1974). Carta Edafológica F13-C69 y F13-C79. México: Instituto Nacional de Estadística Geografía e Informática.

- (2008). Carta Actual de Uso y Vegetación. Serie IV. México: Instituto Nacional de Estadística Geografía e Informática.

- (2010). Geología. Cartas: F13C69 y F13C70. Escala 1:50,000. México: Instituto Nacional de Estadística y Geografía.

- (2010). Instituto Nacional de Estadística y Geografía. Censo de Población y Vivienda. <www.censo2010.org.mx>. [Consulta: 28 de julio de 2013]. 
Lambin, E. y Ehrlich, D. (1997). "The identification of tropical deforestation fronts at broad spatial scales». International Journal of Remote Sensing, 18, 2.568-3.551.

Márquez, R. A. (2008). "Cambio de uso del suelo y el desarrollo turístico en Bahía de Banderas Nayarit». Ciencia UANL, 11 (2), 161-167.

MAS, J. F. (1999). "Monitoring land-cover changes: a comparison of change detection techniques». Internacional Journal of Remote Sensing, 20, 139-152.

MAS, J. F.; DÍAZ, J. R. y PÉREZ, A. (2003). «Evaluación de la confiabilidad temática de mapas o de imágenes clasificadas: una revisión». Investigaciones Geográficas, Boletín del Instituto de Geografía UNAM, 51, 53-72.

Mas, J. F.; VelázQUeZ, A. y Couturier, S. (2009). «La evaluación de los cambios de cobertura/uso del suelo en la República Mexicana». Investigación Ambiental, 1 (1), 23-39.

MendozA, E. y Dirzo, R. (1999). «Deforestation in Lacandonia (southeast México): evidence for the declaration of the northernmost tropical hot-spot». Biodiversity and Conservation, 8, 1.621-1.641.

Morales Hernández, J. C.; Carrillo González, F. M.; Farfán Molina, L. M. y Cornejo López, V. M. (2016). «Cambio de cobertura vegetal en la región de Bahía de Banderas, México». Caldasia, 38 (1), 1.729. $<$ https://dx.doi.org/10.15446/caldasia.v38n1.57831>

Nájera, O.; Bojórquez, J.; Cifuentes J. y Marceleño, S. (2010). "Cambio de cobertura y uso del suelo en la cuenca del río Mololoa, Nayarit». Biociencias, 1 (1), 19-29.

Nene Preciado, A.; González Sansón, G.; Mendoza Cantú, M. y Silva Bátiz, F. (2017). "Cambio de cobertura y uso de suelo en cuencas tropicales costeras del Pacífico Central Mexicano». Investigaciones Geográficas, 0 (94), 1-18. <http://dx.doi.org/10.14350/rig.56770>

OCHOA, S. y GONZÁLEZ, M. (2000). "Land use and deforestation in the highlands of Chiapas, México». Applied Geography, 20 (1), 17-42.

Osorio, L. P.; Mas, J. F.; Guerra, F. y MAass, M. (2015). «Análisis y modelación de los procesos de deforestación: un caso de estudio en la cuenca del río Coyuquilla, Guerrero, México». Investigaciones Geográficas, Boletín del Instituto de Geografía UNAM, 88, 60-74.

Pickett, S. T. A. y Cadenasso, M. L. (2013). «Urban ecology». En: Meyers, Robert A. (ed.). Encyclopedia of Sustainability Science and Technology. Nueva York: Springer, 271-301.

Pinedo, C.; Pinedo, A.; Quintana, A. y Martínez, M. (2007). «Análisis de áreas deforestadas en la región centronorte de la Sierra Madre Occidental, Chihuahua, México». Tecnociencia, 1 (1), 36-43.

Quintero, T. (2004). "Río Cuale, Microcuenca El Cuale, Municipio de Talpa de Allende. SAGARPA-FIRCO». <www.firco.gob.mx/Paginas/default.aspx>. [Consulta: 4 de noviembre de 2012].

RamíReZ, R. I. (2001). "Cambios en las cubiertas del suelo en la Sierra de Angangueo, Michoacán, y Estado de México, 1971-1994-2000». Investigaciones Geográficas, Boletín del Instituto de Geografía UNAM, 45, 39-55.

Reyes, H.; Aguilar, M.; Aguirre, J. y Trejo, I. (2006). "Cambios en la cubierta vegetal y uso del suelo en el área del proyecto Pujal-Coy, San Luis Potosí, México, 1973-2000». Investigaciones Geográficas, Boletín del Instituto de Geografía UNAM, $59,26-42$. 
Rojas, E. G. y OrTiz, I. O. (2009). «Identificación del cilindro nudoso en imágenes TC de trozas podadas de Pinus radiata utilizando el clasificador de máxima verosimilitud». Maderas, Ciencia y Tecnología, 11 (2), 117-127.

Rosete, F. A.; Pérez, J. L. y Bocco, G. (2009). «Contribución al análisis del cambio de uso del suelo y vegetación (1978-2000) en la Península de Baja California, México». Investigación Ambiental, 1 (1), 70-82.

Schawe, M.; Gerold, G.; Bach, K. y Gradstein, S. (2010). "Hydrometeorological patterns in relation to montane forest types along an elevational gradient in the Yungas of Bolivia». En: Bruijnzeel, L.; Scatena, F. y Hamilton, L. (eds.). Tropical Montane Cloud Forests: Science for Conservation and Management, 199-207. Reino Unido: Cambridge University Press.

SEMARNAT (2005). Informe de la situación del medio ambiente en México. <www.semarnat.gob.mx>. [Consulta: 13 de enero de 2014].

TREJO, I. y DiRZO, R. (2000). "Deforestation of seasonally dry tropical forest: a national and local analisis in Mexico». Biological Conservación, 94, 133-142.

TREJO, I. y HERNÁNDEZ, J. (1996). «Identificación de selva baja caducifolia en el estado de Morelos, mediante imágenes de satélite». Investigaciones Geográficas, Boletín del Instituto de Geografía UNAM, 5, 11-18.

USDA (1995). "Guidelines for the use of digital imaginary for vegetation mapping of USA. United States Department of Agriculture». <www.arsusda.gov/rsml/rsmlhome.html>. [Consulta: 16 de mayo de 2013].

Velázquez, A.; Mas, J. F.; Díaz, G.; Mayorga, S.; Alcántara, P. C.; Castro, R.; Fernández, T.; Bocco, G.; Ezcurra, E. y Palacio, J. L. (2002). «Patrones y tasas de cambio de uso del suelo en México». Gaceta Ecológica, Instituto Nacional de Ecología, 62, 21-37.

Weng, Q. (2009). Remote Sensing and GIS Integration: Theories, Methods, and Applications. Nueva York: McGraw-Hill.

WENG, Q. y WEI, Y. D. (2003). "Land use and land cover change in China under the reform and globalization». Asian Geographer, 77 (7), 1-3. 